\title{
When you know that you know and when you think that you know but you don't
}

\author{
EUGENE B. ZECHMEISTER \\ Loyola University of Chicago, Chicago, Illinois 60626 \\ and \\ JOHN J. SHAUGHNESSY \\ Hope College, Holland, Michigan 49423
}

\begin{abstract}
College students rated the likelihood of recall of individual words presented for free recall learning. Predictions were made using a 7-point scale immediately following an item's presentation in the list. To-be-rated items included those presented one time, as well as items presented twice in either a massed (MP) or distributed (DP) manner. Twice-presented items were rated as more likely to be recalled than items presented once, and they were; MP items were judged more likely to be recalled than DP items, but they were not. The finding that subjects think that they know MP items when they do not suggests why processing may be less for massed than for distributed presentations. As such, these results provide support for the attenuation of attention hypothesis of the spacing effect in free recall.
\end{abstract}

When memory for a lengthy list of verbal items is tested, items that have been repeated within the list in a distributed manner (DP) are more likely to be remembered than are items repeated in a massed fashion (MP). As noted by Hintzman (1974), this MP-DP, or spacing, effect has been obtained in a wide variety of memory paradigms, suggesting that it reflects the operation of a fundamental property of the memory system. However, the particular cause of the spacing effect is a matter of significant theoretical dispute (Glenberg, 1979; Hintzman, 1974, 1976).

An explanation of the spacing effect that has received considerable empirical support is the attenuation of attention hypothesis. This explanation was first proposed by Peterson, Wampler, Kirkpatrick, and Saltzman (1963) to account for a spacing effect in a continuous paired associate learning task. The hypothesis makes the seemingly plausible assertion that learners pay less attention to massed repetitions than to distributed repetitions of a to-be-remembered item because massed repetitions occur in close proximity to the item's initial presentation. The hypothesis is supported by the finding that subjects spend less time studying massed presentations of an item than studying distributed presentations when study is self-paced (Shaughnessy, Zimmerman, \& Underwood, 1972). Further indirect evidence in support of the hypothesis is provided by the finding that performance on a reaction time task carried out concurrently

This research was reported at the 15 th annual meeting of the Psychonomic Society, 1974. Requests for reprints should be sent to Eugene B. Zechmeister, Psychology Department, Loyola University of Chicago, 6525 N. Sheridan Road, Chicago, Illinois 60626 . with list processing is better during massed than during spaced presentations of list items, indicating that subjects devote less processing capacity to MP than to DP items (Johnston \& Uhl, 1976). However, Hintzman (1974) has criticized the attention hypothesis because "such an account of the spacing effect should say why the subject chooses not to attend to $\mathrm{P} 2$ at short spacings" (p. 89). The present research was done to examine one possible basis for subjects' attenuation of attention.

It is possible that the subjects do not attend to massed presentations of a list item because they mistakenly believe the item has been processed well enough to be remembered on a later test. In other words, subjects think that they know it but they do not. Jacoby (1978) has recently likened the task of learning a list of verbal items to that of solving a series of problems. For each item the problem is how to get the item successfully encoded. When repetitions of an item are massed, subjects may be misled into thinking that the encoding problem was solved during the initial presentation of the item and that further work on this item is not required. The ease with which information about the item can be retrieved at the time of its massed repetition may lead the subject to have undue confidence in the item's memory strength. If this overconfidence does occur, it could explain why motivational inducements such as paying subjects extra money to remember MP items have failed to eliminate the spacing effect (Hintzman, Summers, Eki, \& Moore, 1975). If subjects believe that they already know a MP item, they may see no reason for additional processing no matter what the payoff (Shaughnessy, 1976). 
In the present experiment, subjects were asked to make predictions regarding their memory for individual items while studying a list of to-be-remembered words. That is, immediately after studying an item, subjects judged whether they knew the item well enough to recall it on a later test. Predictions were required for half the list items, some of which had been presented only one time and some of which had been presented twice in either a massed or distributed fashion. It was expected that estimates of recallability would be higher for repeated items than for once-presented items. More critically, however, it was expected that the highest predictions would be those for MP items.

\section{METHOD}

\section{Lists}

The to-be-remembered items were common nouns randomly selected from the list prepared by Spreen and Schulz (1966). All words had more than four letters; equal numbers of one-, two-, and three-syllable words were used. Each experimental list included 24 critical items arranged in four study blocks. In each block there were six items, two appearing one time (singles) and four appearing twice. Two of the four twice-presented items were MP items and two were DP items. The lag between presentation of the DP items was varied nonsystematically with 3,4 , or 5 items between the presentation of a given distributed item. Nouns were randomly assigned to study blocks, with the restriction that an equal number of words of each syllable length appear in each block. Particular words within a study block served equally often (once) as each of the six study items. Therefore, there were six experimental lists. A primacy buffer of four items, two singles, one MP, and one DP item, was also used in each list. All study list words were typed on 2 by 2 slides.

\section{Procedure}

The experiment was introduced to subjects as one involving free recall of common words. Subjects were informed that the items would be presented singly for several seconds on a screen in front of them. They were also informed that some items would appear more than one time and that the experimenter was interested in their memory for the complete list of words.

In addition, subjects were told that for some words in the list they would be asked to rate the likelihood that they would recall these items on a later recall test. It was suggested that this kind of judgment was not unlike that required when they were preparing for a class examination. That is, they were to assess how well they knew something before they were actually tested. Subjects were told that whenever the screen was blank, they were to use a 7-point rating scale to indicate how sure they were that the item they had just studied would be recalled on the later test. Low numbers on the 7-point rating scale were used to indicate a low probability of recall; higher numbers were to be used to indicate increasing certainty of later recall. Ratings of twicepresented items were always made following the second occurrence of the item. Not all the items were to be rated, and subjects were informed that the experimenter was interested in their memory for all list items, including those not rated. In fact, half the items in each study block (one of each item type) were rated by subjects. Two items in the primacy buffer were also rated. Subjects recorded their ratings on an answer sheet that showed the rating scale at the top of the page and contained spaces for 42 ratings, although fewer than half that number were required in the experiment. Additional spaces were included so that subjects would not know when the end of the list was approaching. Prior to presentation of the word list, all subjects were briefly practiced on the rating task using a short list of three-digit numbers.
Study list items were presented at a 6 -sec rate, and $6 \mathrm{sec}$ were also allowed for subjects to make their ratings. A 1-min math test was given to all subjects after the presentation of the last list item in order to control for recency effects. Four minutes were allowed for free recall.

Twenty-four students at Loyola University of Chicago were subjects. Participation was part of an introductory psychology course requirement. All subjects were tested individually, with four subjects assigned via a block randomization procedure to each of the six experimental lists.

\section{RESULTS}

Proportion recall for the three item types and for both rated and nonrated items is shown in Figure 1. A typical spacing effect was obtained for free recall of twice-presented items $[\mathrm{F}(1,23)=5.9, \mathrm{MSe}=.82, \mathrm{p}<$ .05]. Further, the difference in recall between MP and DP items was similar for both rated and nonrated items, the interaction between conditions of spacing and rating not being significant $(F<1)$. Thus, the requirement to rate items during free recall did not alter the usual recall effect associated with presentation of MP and DP items. The overall level of recall of rated items was, however, significantly higher than that of nonrated items $[\mathrm{F}(1,23)=6.0, \mathrm{MSe}=.82, \mathrm{p}<.05]$. It must be remembered that potential study time for rated items exceeded that for nonrated items due to the fact that subjects made their ratings during a 6-sec interval following each to-be-rated item. In fact, when single items were studied and then rated, effective study time was potentially double that for nonrated items.

Ratings of recall likelihood for the three different item types are shown in Table 1. Looking first at overall mean ratings, it can be seen that estimates of recallability were greater for twice-presented items, either MP or DP, than for singles. Given that recall of these items

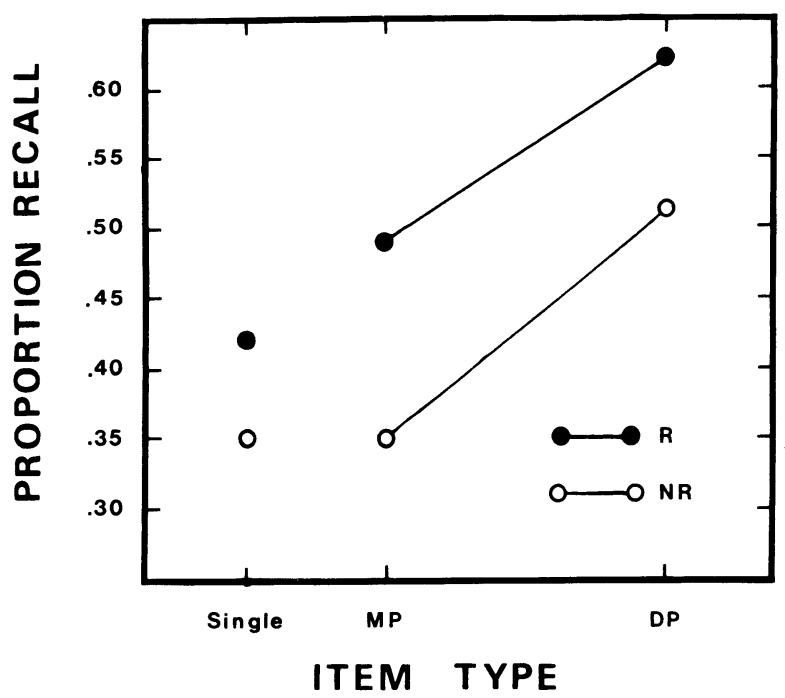

Figure 1. Proportion recall of once-presented (single) and twice-presented (MP and DP) items that were either rated (R) or not rated (NR) for likelihood of recall. 
differed in the same direction, subjects can be said to have accurately assessed the probability of recall to be higher for an item presented twice than for an item presented one time. A t test of the difference in mean ratings provided for DP and single items was statistically significant $\left[\mathrm{t}(23)=4.06, \mathrm{~S}_{\text {diff }}=.25, \mathrm{p}<.05\right]$.

When mean ratings of likelihood of recall are examined for MP and DP items, the results shown in Table 1 reveal that in each of the four study blocks ratings associated with MP items were greater than those for DP items. The MP items were consistently judged to be more recallable than the DP items. However, the overall mean difference between ratings for these two item types was not statistically significant $[\mathrm{t}(23)=1.40]$. Nonetheless, relative to recall of these two kinds of twice-presented items, ratings for MP and DP items were in the direction predicted. The relative difference between recall probability and mean rating of likelihood of recall is illustrated in Figure 2, which describes both rating and recall performance in terms of $\mathrm{z}$ scores. Recall performance summarized in Figure 2 is based on the combined performance of rated and nonrated items, but similar results are obtained if only rated items are scored for recall. The pattern of results seen in Figure 2 clearly

Table 1

Mean Rating and Proportion Recall for Items Rated for Likelihood of Recall in Each of Four Study Blocks

\begin{tabular}{llrrrrr}
\hline \multirow{2}{*}{$\begin{array}{l}\text { Item } \\
\text { Type }\end{array}$} & Measure & \multicolumn{1}{c}{1} & \multicolumn{1}{c}{ Block } & \multicolumn{1}{c}{$\begin{array}{c}\text { Over- } \\
\text { all }\end{array}$} \\
\cline { 3 - 6 } DP & Rating & 4.50 & 4.29 & 4.00 & 4.12 & 4.23 \\
& Recall & .50 & .54 & .58 & .88 & .62 \\
\multirow{2}{*}{ MP } & Rating & 4.88 & 4.33 & 4.29 & 4.83 & 4.57 \\
& Recall & .38 & .42 & .46 & .71 & .49 \\
\multirow{2}{*}{ Single } & Rating & 3.42 & 3.04 & 3.21 & 3.33 & 3.25 \\
& Recall & .25 & .46 & .33 & .62 & .42 \\
\hline
\end{tabular}

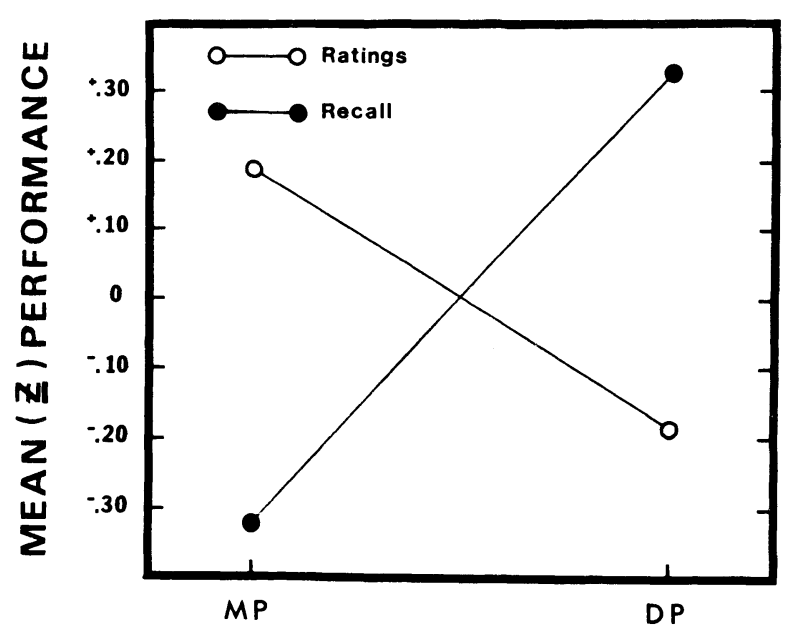

ITEM TYPE

Figure 2. Mean rating and recall for MP and DP items when both rating and recall performance are expressed in $\mathrm{z}$ scores. shows that the difference between ratings of likelihood of recall for MP and DP items is in marked opposition to these items' recall probability. Using $\mathrm{z}$ scores, the average MP-DP difference in recall performance was contrasted with that for the rating task, and the resultant difference of the differences was statistically significant $\left[\mathrm{t}(23)=2.64, \mathrm{~S}_{\text {diff }}=.39, \mathrm{p}<.05\right]$.

A final analysis was done to examine recall of items as a function of subjects' mean ratings of recall likelihood. For all three item types, recall probability was greater for items given a high rating than for items given low or intermediate ratings. The proportion recall for items receiving a low likelihood rating ( 1 or 2$)$, an intermediate rating $(3,4$, or 5$)$, or a high prediction of recall (6 or 7) was as follows for the three types of items: singles, . $33, .35, .67$; MP, $.33, .31, .59$; DP, $.38, .47, .73$. Therefore, for the various types of items, subjects showed a similar ability to identify those items that were most likely to be recalled.

\section{DISCUSSION}

As an explanation for the spacing effect, the attenuation of attention hypothesis has been found wanting because it fails to identify the reason for subjects' lack of attention to massed presentations of to-be-remembered items. The rationale for the present study was based on the assumption that this lack of attention is the result of subjects' tendencies to overestimate their ability to remember an item that has been presented under MP conditions. To the extent that subjects believe they have successfully encoded an item, it can be assumed that little in the way of further processing will be attempted. This explanation for the attention deficit was tested by asking subjects to predict at the time of study the likelihood of recall of individual items. Mean ratings of recall likelihood were found to be greater for MP than for DP items, even though recall differed significantly in the opposite direction. At the time of encoding, subjects apparently believe MP items are more likely to be recalled than are DP items.

Although the present argument has emphasized the overestimation of recall likelihood for MP items, the results are equally consistent with processes that would result in underestimation of recall likelihood for DP items. For example, perhaps the DP schedule serves to alert subjects to the fragility of their memory for these items. The most critical aspect of the present findings, however, is that ratings of recall likelihood differ for MP and DP items. As such, these different judgments may serve as the basis of subjects' decisions to process MP and DP items differently, and it is this differential processing that is the source of the spacing effect according to the attenuation of attention hypothesis.

The present experiment also demonstrated that learners can accurately evaluate the future memorability of presently studied information. Twice-presented items were given higher estimates of recall probability and were indeed more likely to be recalled than were once-presented items. Also, across the several item types, those items identified by subjects as most likely to be recalled were in fact recalled with a greater probability than those given lower ratings. This aspect of the learner's memorymonitoring ability has been relatively neglected in the current wave of interest in topics of metamemory (but see Arbuckle \& Cuddy, 1969; Groninger, 1979). The ability to evaluate what is known and what is not known at time of study would appear to be critical for efficient study. Without knowing what material has been successfully encoded, a learner cannot allot study time such that less well learned items receive more attention than those that are already well learned. 
The present results do not allow specification of the basis of subjects' memory-monitoring accuracy, but they suggest that it is important to identify those situations that are likely to lead learners to believe that material is well learned when in fact it is not. Few instructors have not been confronted with the student who, having done poorly on an examination, offers the excuse, "I thought I really knew the material." Such erroneous impressions may be related to undue emphasis on certain characteristics of the student's study routine. For example, a tendency to equate time spent studying with amount learned may be fatal scholastically if processing during that time is inadequate for permanent memory registration. In general, further study is needed to determine the factors that facilitate and retard accuracy of judging what is known and what is not known within recently studied information.

\section{REFERENCES}

Arbuckle, T. Y., \& Cuddy, L. L. Discrimination of item strength at time of presentation. Journal of Experimental Psychology, 1969, 81, 126-131.

Glenberg, A. M. Component-levels theory of the effects of spacing of repetitions on recall and recognition. Memory \& Cognition, 1979, 7, 95-112.

Groninger, L. D. Predicting recall: The "feeling-that-I-willknow" phenomenon. American Journal of Psychology, 1979, 92, 45-58.

Hintzman, D. L. Theoretical implications of the spacing effect. In R. L. Solso (Ed.), Theories in cognitive psychology: The Loyola symposium. Hillsdale, N.J: Erlbaum, 1974.
Hintzman, D. L. Repetition and memory. In G. H. Bower (Ed.), The psychology of learning and motivation (Vol. 10). New York: Academic Press, 1976.

Hintzman, D. L., Summers, J. J., Eki, N. T., \& Moore, M. D. Voluntary attention and the spacing effect. Memory \& Cognition, 1975, 3, 576-580.

JACOBY, L. L. On interpreting the effects of repetition: Solving a problem versus remembering a solution. Journal of Verbal Learning and Verbal Behavior, 1978, 17, 649-667.

Johnston, W. A., \& UhL, C. N. The contribution of encoding effort and variability to the spacing effect on free recall. Journal of Experimerial Psychology: Human Learning and Memory, 1976, 2, 153-160.

Peterson, L. R., Wampler, R., Kirkpatrick, M., \& Saltzman, D. Effect of spacing presentations on retention of a paired associate over short intervals. Journal of Experimental Psychology, 1963, 66, 206-209.

Shaughnessy, J. J. Persistence of the spacing effect in free recall under varying incidental learning conditions. Memory \& Cognition, 1976, 4, 369-377.

Shaughnessy, J. J., Zimmerman, J., \& Underwood, B. J. Further evidence on the MP-DP effect in free-recall learning. Journal of Verbal Learning and Verbal Behavior, 1972, 11, $1-12$.

Spreen, O., \& Schulz, R. W. Parameters of abstraction, meaningfulness, and pronunciability for 329 nouns. Journal of Verbal Learning and Verbal Behavior, 1966, 5, 459-468.

(Received for publication October 24, 1979.) 